\title{
Analysis of conditions for synthesis of CdS:Mn nanoparticles
}

\author{
A.O. Kovalchuk ${ }^{1}$, G.Yu. Rudko ${ }^{1}$, V.I. Fediv ${ }^{2}$, E.G. Gule ${ }^{1}$ \\ ${ }^{I} V$. Lashkaryov Institute of Semiconductor Physics, National Academy of Sciences of Ukraine, \\ 45, prospect Nauky, 03028 Kyiv, Ukraine \\ ${ }^{2}$ Bukovinian State Medical University, Department of Biophysics and Medical Informatics, \\ 42, Kobylyanska str., 58000 Chernivtsi, Ukraine \\ Corresponding author phone: +38(097)669-41-74, e-mail: andriy_kovalchuk@yahoo.com
}

\begin{abstract}
The detailed analysis of the synthesis of CdS:Mn nanoparticles in polyvinyl alcohol polymeric matrix is presented and the ranges of optimal growth parameters are determined. The estimated values of parameters were used to produce solutions of CdS:Mn nanoparticles and corresponding solid composites. The sizes of nanoparticles and structure of composite films were characterized by electron microscopy methods, optical properties were probed using photoluminescence methods.
\end{abstract}

Keywords: nanocomposite, nanoparticles, CdS, polymer, synthesis.

Manuscript received 17.09.14; revised version received 29.12.14; accepted for publication 19.02.15; published online 26.02.15.

\section{Introduction}

Nanoparticles (NP) and nanoparticle-based composites were in the focus of solid-state researches for the last decades [1]. NPs offer a wide range of applications such as light-emitting diodes [2], photocatalysts [3], biological labels [4], electrochemical cells [5], lasers [6], etc. In most of these applications, NPs were synthesized using the colloidal chemistry methods that were proved to be inexpensive and comparatively simple. By using these methods, one can produce solutions of NPs, nanopowders or even nanocomposite films that can be cast directly from the reaction solutions. Among the latter, the hybrids of semiconductor NPs with polymers are supposed to be the materials of great future. Polymers are considered to be a good choice as matrix materials for incorporating NPs due to their stability, flexibility and transparency in the visible range. Incorporation of NPs into polymeric matrices adds new properties to the hybrid such as size-dependent as well as doping-dependent absorption and emission.
For the synthesis to be successful and reproducible, a clear understanding of the processes occurring in the growth solution and the conditions for their realization is of utmost importance. However, despite a wide variety of implementations of the colloidal synthesis route, at present, there is no general recipe for choosing the parameters of synthesis. In view of the challenges that lie in the understanding of optimum conditions for NP growth, we herein report a theoretical analysis of chemical processes in the growth solution in order to optimize the conditions of synthesis. We have focused on the synthesis of CdS:Mn nanoparticles in the solution of polyvinyl alcohol. The goal of the study is to find out the ranges of growth parameters variation that provide optimum conditions for NP fabrication. We also apply the predicted parameters to grow NPs in-situ in solution of polymer, use this solution to create thin nanocomposite films and carry out the characterization of the nanocomposite obtained in this manner by using electron microscopy and optical methods. 


\section{Experimental}

\subsection{Synthesis procedure}

Composites of CdS:Mn nanoparticles were synthesized via colloidal chemical route. The synthesis process took place in $5 \%$ aqueous solution of PVA. Here, PVA plays a double role: firstly, the macromolecules serve as a capping agent for NPs during the growing procedure; secondly, PVA is a suitable polymeric matrix for formation of free-standing films of NP-containing composite. The precursors for NP growth were sodium sulfide, cadmium chloride and manganese chloride. Optimized values of the concentrations of these precursors as well as $\mathrm{pH}$ of the solution will be estimated in Section 3. Thin solid films of the nanocomposite were obtained by drying the solution in a closed vessel containing an absorbent. All the synthesis steps and drying process were performed under ambient conditions.

\subsection{Characterization techniques}

$\mathrm{NP}$ formation in colloids was probed using the transmission electron microscopy method with JEM100SX electron microscope. The accelerating voltage value was $100 \mathrm{keV}$. The samples for micrograph imaging were prepared by drying a droplet of the NP-containing solution on the copper grating. The spatial resolution of the microscopic image was $0.7 \mathrm{~nm}$.

Optical properties of nanocomposites were characterized using the photoluminescence (PL) technique. To measure PL spectra, we used MDR-23 grating spectrometer. The LED with $\lambda=375 \mathrm{~nm}$ was used as an excitation source. PMT-100 was used for detection in the visible spectral range and PMT-62 was used for IR detection.

\section{Analysis of chemical reactions probabilities during NP synthesis for optimization of synthesis conditions}

Formation of NPs with targeted properties in the solutions containing capping molecules depends on many parameters, namely: precursors, solvents and capping molecules concentrations in the growth solution, $\mathrm{pH}$ of the growth medium, temperature, pressure, etc. In the present study aimed at growing NPs under ambient conditions, we have focused on the thorough analysis of the influence of absolute and relative concentrations of the growth solution components as well as $\mathrm{pH}$ values on successful production of doped NPs. The goal of these parameters values adjustment is, on the one hand, to provide successful NPs formation, and on the other hand, to eliminate formation of unwanted compounds. For this purpose, we have done theoretical calculations of chemical reactions probabilities for synthesizing NPs in multicomponent polymer-salts systems.

\subsection{Analysis of the products of salts hydrolysis}

One of the important factors that control NPs formation is the $\mathrm{pH}$ value of the growth medium. It strongly influences the relative content of various ions in solution. Due to dissociation and hydrolysis reactions, the following ions and neutral species can be found in the growth solution:

1) the products of $\mathrm{Na}_{2} \mathrm{~S}$ dissociation -ions $\mathrm{Na}^{+}, \mathrm{S}^{2-}$. The subsequent hydrolysis in water produces also $\mathrm{H}_{2} \mathrm{~S}, \mathrm{HS}^{-}, \mathrm{S}^{2-}$;

2) the products of $\mathrm{MnCl}_{2}$ dissociation - ions $\mathrm{Mn}^{2+}, \mathrm{Cl}^{-}$. Further hydrolysis leads to the formation of $\mathrm{Mn}(\mathrm{OH})^{+}, \mathrm{Mn}(\mathrm{OH})_{2}{ }^{0}$;

3) the products of $\mathrm{CdCl}_{2}$ dissociation - ions $\mathrm{Cd}^{2+}, \mathrm{Cl}^{-}$, and, respectively, the products of hydrolysis $\mathrm{Cd}(\mathrm{OH})^{+}, \mathrm{Cd}(\mathrm{OH})_{2}{ }^{0}, \mathrm{Cd}(\mathrm{OH})_{3}{ }^{-}$.

To calculate the mole fractions of the hydrolysis products in the growth solution $v s$. $\mathrm{pH}$, we have analyzed the material balance equations for cadmium, manganese and sulphur, and have plotted the diagrams of the hydrolysis products content $v s$. pH. Fig. 1 illustrates the presence of the above-listed products of hydrolysis in the growth solution. The upper, middle and lower panels represent the regions where one of the species listed in (1), (2) or (3), respectively, dominates (i.e. the relative content of this specific ion exceeds $50 \%$ ) in the growth solution. As it follows from the upper panel, within the $\mathrm{pH}$ range from 0 to 13 , the dominating species in the solution are $\left(\mathrm{H}_{2} \mathrm{~S}+\mathrm{HS}^{-}\right)$ions, while at higher $\mathrm{pH}$ values, the relative concentration of $\mathrm{S}^{2-}$ exceeds $50 \%$. Similarly, the middle panel shows that within the $\mathrm{pH}$ range from 0 to $10.5 \mathrm{Mn}^{2+}$ ions dominate in the solution, within the $\mathrm{pH}$ range from 10.5 to 12.3 $\mathrm{Mn}(\mathrm{OH})^{+}$ions dominate, and within the $\mathrm{pH}$ range from 12.3 to $16 \mathrm{Mn}(\mathrm{OH})_{2}^{0}$ species dominate.

Based on the results mapped in Fig. 1, one can conclude that within the range of $\mathrm{pH}$ values from 0 to 7.5 the relative content of $\mathrm{Cd}^{2+}, \mathrm{Mn}^{2+}, \mathrm{HS}^{-}$among the hydrolysis products simultaneously exceeds $50 \%$. To secure the best growth conditions, we have chosen for our synthesis the much narrower range from 3.5 to 6.5 ; it is shaded with grey colour in Fig. 1.

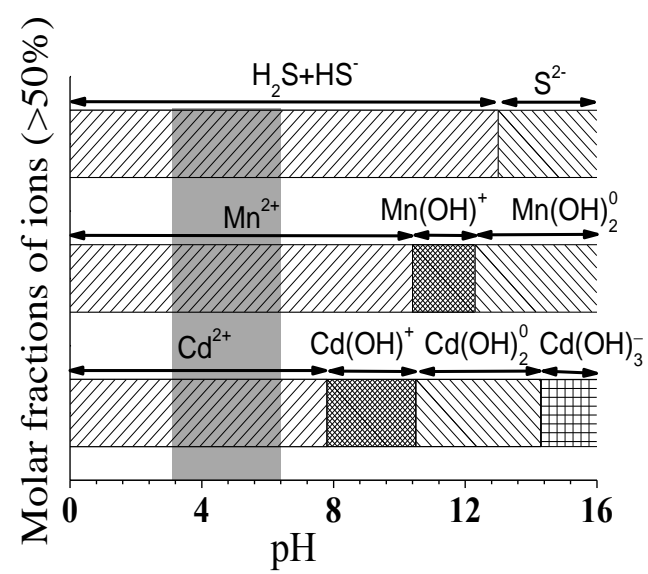

Fig. 1. Diagram that illustrates the relative content of the products of salts hydrolysis in the solution at varied $\mathrm{pH}$. Differently hatched rectangles represent the regions where the labelled ions dominate in the growth solution (i.e., where the relative concentration of ions exceeds $50 \%$ ). 


\subsection{Analysis of relative concentrations the products of complexation involving $\mathrm{Cd}^{2+}$ and $\mathrm{Mn}^{2+}$}

It is known [7] that, besides the hydrolysis products, chlorine-containing complexes of the type $\left[\mathrm{MeCl}_{n}\right]^{2-n}$ can be formed in water solutions of $\mathrm{MeCl}_{2}$ salts. We have carried out the material balance equation analysis [8] taking into account the stability constants of chlorine complexes with cadmium and manganese $[9,10]$. The calculations were done for the $\mathrm{pH} 3$ to 7 range. The results of this analysis are presented in Fig. 2 that schematically depicts the regions where the complexes dominate in different $\mathrm{pCl}$ ranges. It is seen that the region $\mathrm{Mn}^{2+}$ ions domination (concentration >50\%) extends up to the salt concentration $0.1 \mathrm{~mol} / \mathrm{dm}^{3}$, while the similar region for $\mathrm{Cd}^{2+}$ ions is limited by the concentration $0,05 \mathrm{~mol} / \mathrm{dm}^{3}$. Based on the results mapped in Fig. 2, one can conclude that the optimal pCl values exceed 2 (as shown by grey shade in Fig. 2), which corresponds to the salt concentrations less than $0.05 \mathrm{~mol} / \mathrm{dm}^{3}$.

\subsection{Analysis of the probabilities of $\mathrm{Cd}(\mathrm{OH})_{2}, \mathrm{Mn}(\mathrm{OH})$, $\mathrm{CdS}$ and $\mathrm{MnS}$ precipitation}

Among other possible reactions, the probability of $\mathrm{Cd}(\mathrm{OH})_{2}$ and $\mathrm{Mn}(\mathrm{OH})_{2}$ hydroxides formation is substantial. Hydroxides precipitation is an obstructive effect for chemical synthesis of sulfides; thus, it is important to find out the ranges of synthesis parameters where these processes are suppressed. To analyze the probability of cadmium and manganese hydroxide precipitation, we have applied the procedure proposed in [11] and solved the corresponding equations taking into account the solubility products of $\mathrm{Cd}(\mathrm{OH})_{2}$ and $\mathrm{Mn}(\mathrm{OH})_{2}$. Analogously, to analyze the probability of $\mathrm{CdS}$ and $\mathrm{MnS}$ formation, we have also used the procedure proposed in [11] keeping in mind the solubility products of these salts.

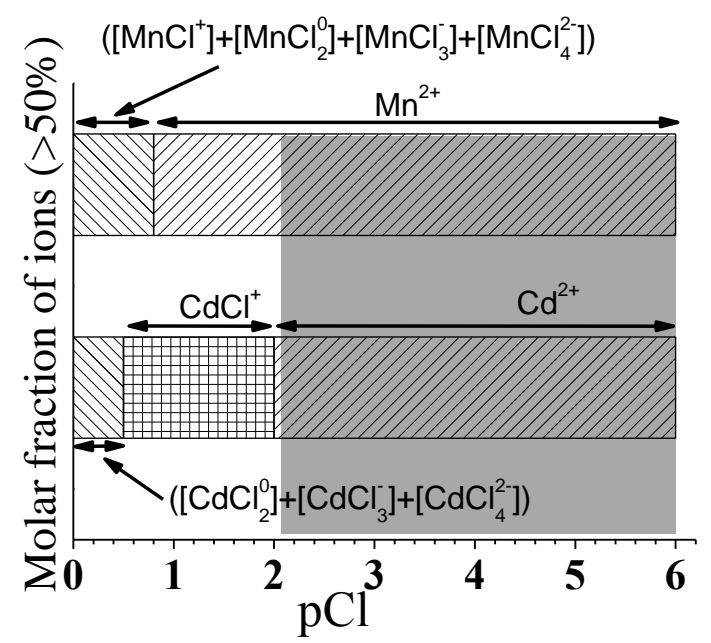

Fig. 2. Schematic representation of the regions of the $\mathrm{Cl}^{-}$ions concentration where respective complexation products dominate.

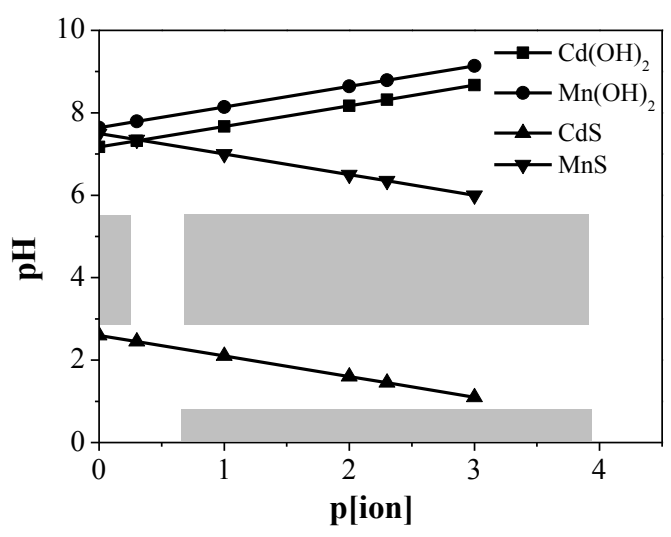

Fig. 3. The dependences of the $\mathrm{pH}$ values at which precipitation of $\mathrm{Cd}(\mathrm{OH})_{2}, \mathrm{Mn}(\mathrm{OH}), \mathrm{CdS}$ and $\mathrm{MnS}$ starts (curves 1-4, respectively). Grey color indicates the range of $\mathrm{pH}$ where the probabilities of CdS precipitation are high and, simultaneously, the probabilities of $\mathrm{MnS}, \mathrm{Cd}(\mathrm{OH})_{2}$ and $\mathrm{Mn}(\mathrm{OH})_{2}$ precipitation are low.

Fig. 3 shows the dependences of the $\mathrm{pH}$ values, at which precipitation of $\mathrm{Cd}(\mathrm{OH})_{2}, \mathrm{Mn}(\mathrm{OH})_{2}, \mathrm{CdS}$ and $\mathrm{MnS}$ starts, vs. salts concentrations. From this plot the optimum $\mathrm{pH}$ values for $\mathrm{NP}$ growth were chosen. On the one hand, as it is seen from the plot, precipitation of $\mathrm{Cd}(\mathrm{OH})_{2}$ and $\mathrm{Mn}(\mathrm{OH})_{2}$ occurs at $\mathrm{pH}>7$. Therefore, to avoid formation of hydroxides in the system $\mathrm{MeCl}_{2}$ $\mathrm{Na}_{2} \mathrm{~S}-\mathrm{H}_{2} \mathrm{O}$, one has to choose $\mathrm{pH}<7$. On the other hand, precipitation of $\mathrm{CdS}$ starts at $\mathrm{pH}>2$, while precipitation of $\mathrm{MnS}$ starts at $\mathrm{pH}>6$. Therefore, to achieve formation CdS:Mn NPs and simultaneously avoiding MnS precipitation, one has to choose $\mathrm{pH}$ values within the range 2 to 6 . To secure the best synthesis conditions, we have chosen the $\mathrm{pH}$ range 3 to 5 , which is indicated with grey color in Fig. 3.

\section{Characterization of NPs and nanocomposites synthesized using the procedure with the parameters predicted}

\subsection{TEM results}

All the growth conditions that were estimated in the previous Section were applied to synthesize CdS NPs in the PVA solution. The obtained colloidal solution was studied by using the transmission electron spectroscopy method. The typical TEM photo of NPs washed out of the growth solution is shown in Fig. 6. This microscopic image allowed estimating the average radius of NPs that lies within the range 5 to $10 \mathrm{~nm}$.

\subsection{Photoluminescence studies}

Optical properties of the nanocomposites obtained from the colloidal solutions were studied using the photoluminescence method. Fig. 5 illustrates the influence of $\mathrm{pH}$ of the solution on the radiative properties of the CdS:Mn/PVA nanocomposite. The curve represents the ratio between the intensities of high- 
and low-energy bands in the PL spectrum (see the insert) $v s$. the $\mathrm{pH}$ value of the synthesis solution. The example of the PL spectrum shown in the insert corresponds to the CdS:Mn/PVA composite sample synthesized at $\mathrm{pH}=5.2$. The shape of this specific spectrum is typical for CdS:Mn/PVA nanocomposites obtained at all other $\mathrm{pH}$ values used. All the spectra studied consist of two wide bands: one is in the blue-green region with the maximum at $475 \mathrm{~nm}$, the other one is in the yellow-red region with the maximum at $600 \mathrm{~nm}$. Similar bands were previously observed in doped colloidal CdS NPs grown by somewhat different growing procedures. The highenergy band lies in the spectral range of the near band edge luminescence and is usually ascribed to shallow traps $[12,13]$. The low-energy band was interpreted in two ways. The radiative transitions in this spectral range were either ascribed to some deep traps formed by the surface defects in doped CdS NPs [12, 14-16] or to intra$3 \mathrm{~d}$ transition of $\mathrm{Mn}^{2+}$ ions incorporated in CdS crystals $[14,17,18]$. We assume that both types of radiative centers contribute to the low-energy band observed in our samples [18]. Thus, we have fitted the spectra with three components. The dotted lines in Fig. 5 (insert) show the results of fitting the PL bands.

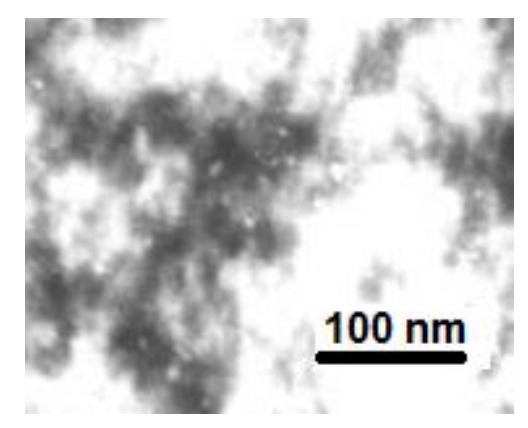

Fig. 4. TEM photo of CdS:Mn NPs.



Fig. 5. The ratio between intensities of high and low energy PL bands vs. the $\mathrm{pH}$ value of synthesis solution. The inset: PL spectra of composite synthesized at $\mathrm{pH}=5.2$ in the visible range. Dotted lines show the results of the spectra fitting. $\mathrm{T}=300 \mathrm{~K} ; \lambda_{\text {exc }}=375 \mathrm{~nm}$.
As it is seen from Fig. 5, the relative intensity of the complex low-energy PL band is reduced as compared to the high-energy band with decreasing the $\mathrm{pH}$ value. This can imply that increasing the $\mathrm{pH}$ value can lead to increasing the number of surface-related radiative recombination centers due to adsorption of $\mathrm{OH}$-ions on the surface of CdS:Mn nanoparticles and/or to the increase of the $\mathrm{Mn}$-related radiative centers number [18].

\section{Conclusions}

We have reported the results of the analysis of chemical reactions probabilities aimed at optimizing the conditions of synthesis of CdS:Mn nanoparticles in the PVA solution. The estimated ranges of the growth parameters are as follows: optimal $\mathrm{pH}$ range -3 to 5 , optimal initial salts concentrations $<0.05 \mathrm{~mol} / \mathrm{dm}^{3}$.

The optimized parameters were applied to grow NPs in-situ in the solution of polymer. The electron microscopy studies have confirmed successful formation of NPs. The obtained solutions of NPs were feasible for formation of thin nanocomposite films of good homogeneity.

\section{References}

1. N. Gaponik, S.G. Hickey, D. Dorfs, A.L. Rogach, and A. Eychmuller, Progress in the light emission of colloidal semiconductor nanocrystals // Small, 6(13), p. 1364-1378 (2010).

2. J. Zhao, J.A. Bardecker, A.M. Munro, M.S. Liu, Y.H. Niu, I.K. Ding, J.D. Luo, B.Q. Chen, A.K.Y. Jen, D.S. Ginger, Efficient CdSe/CdS quantum dot light-emitting diodes using a thermally polymerized hole transport // Nano Lett. 6, p. 463-467 (2006).

3. A. Kubacka, B. Bachiller-Baeza, G. Colyn, M. Fernandez-Garcha, W, N-co-doped $\mathrm{TiO}_{2}$ anatase: a sunlight-operated catalyst for efficient and selective aromatic hydrocarbons photo-oxidation // J. Phys. Chem. C, 113, p. 8553-8555 (2009).

4. M. Bruchez, M. Moronne, P. Gin, S. Weiss, A.P. Alivisatos, Semiconductor nanocrystals as fluorescent biological labels // Science, 281, p. 2013-2016 (1998).

5. L. Li, W. Wang, H. Liu, X. Liu, Q. Song, S. Ren, First principles calculations of electronic band structure and optical properties of Cr-doped $\mathrm{ZnO} / /$ J. Phys. Chem. C, 113, p. 8460-8464 (2009).

6. Y.J. Chan, S. Steckel, P.T. Snee, J.M. Caruge, J.M. Hodgkiss, D.G. Nocera, M.G. Bawendi, Blue semiconductor nanocrystal laser // Appl. Phys. Lett. 86, 073102 (2005).

7. I. Fishtyk, Thermodynamics of Complex Chemical Equilibria. Shtynista, Chisinau, 1989.

8. J. Butler, Ionic Equilibrium: A Mathematical Approach. Reading, MA: Addison-Wesley, 1964. 
9. V.S. Kulbanovsky, Complexes of manganese chloride (II) in the system: $\mathrm{MnCl}_{2}-\mathrm{NH}_{4} \mathrm{Cl}-\mathrm{H}_{2} \mathrm{O} / /$ Zhurnal neorganich. khimii, 22(3), p. 735-738 (1977), in Russian.

10. K.B. Yacemirsky, V.P. Vasiliev, Instability Constants of Complex Compounds. Publ. Academy of Science of USSR, Moscow, 1959 (in Russian).

11. B.P. Nikolsky, Handbook of Chemist. Chemistry, Moscow, 1965 (in Russian).

12. P.K. Khanna, R.R. Gokhale, V.S. Subbarao, N. Singh, K.-W. Jun, B.K. Das, Synthesis and optical properties of CdS/PVA nanocomposites // Mat. Chem. Phys. 94, p. 454-459 (2005).

13. M. Tanaka, Y. Masumoto, Energy transfer mechanism in $\mathrm{Mn}^{2+}$ doped CdS nanocrystals // Solid State Communs. 120, p. 7-10 (2001).

14. M. Tanaka, J. Qi, Y. Musumoto, Optical properties of undoped and $\mathrm{Mn}^{2+}$-doped $\mathrm{CdS}$ nanocrystals in polymer // J. Cryst. Growth. 214, p. 410-414 (2000).
15. J.C. Ferrer, A. Salinas-Castillo, J.L. Alonso, S. Fernandez, R. Mallavi, Influence of SPP costabilizer on the optical properties of CdS quantum dots grown in PVA // Physics Procedia, 2, p. 335338 (2009).

16. D. Kim, M. Miyamoto, M. Nakayama, Surfacemodification effects on luminescence properties of $\mathrm{CdS}$ and CdMnS quantum dots prepared by a reverse-micelle method // phys. status solidi $(c), \mathbf{4}$, p. 1233-1236 (2003).

17. A. Ishizumi, Y. Kanemitsu, Luminescence spectra and dynamics of Mn-doped CdS core/shell nanocrystals // Adv. Mater. 18, p. 1083-1085 (2006).

18. V.I. Fediv, G.Yu. Rudko, A.I. Savchuk, E.G. Gule, A.G. Voloshchuk, Synthesis route and optical characterization of CdS:Mn/polyvinyl alcohol nanocomposite // Semiconductor Physics, Quantum Electronics \& Optoelectronics, 15(2) p. 117-123 (2012). 